Tropical Journal of Pharmaceutical Research December 2018; 17 (12): 2477-2481

ISSN: 1596-5996 (print); 1596-9827 (electronic) (1) Pharmacotherapy Group, Faculty of Pharmacy, University of Benin, Benin City, 300001 Nigeria.

\title{
Effect of edaravone-urinary kallidinogenase combination treatment on acute cerebral infarction
}

\author{
Xin-Min Wang ${ }^{1 *}$, Li Zhu', Ying-Jun Sun ${ }^{2}$, Xiao-Ying Liu', Shu-Gang Dong ${ }^{1}$, Jing \\ Han $^{2}$, Jin-Ming $\mathrm{Ji}^{1}$ \\ ${ }^{1}$ Department of Neurology, ${ }^{2}$ Department of Endocrinology, Binzhou People's Hospital, Binzhou, Shandong, 256610, PR China \\ *For correspondence: Email: wangxinminbb@163.com; Tel: +86-0543-3282291
}

Sent for review: 3 May 2018

Revised accepted: 21 November 2018

\begin{abstract}
Purpose: To investigate the curative effect of edaravone in combination with urinary kallidinogenase in the treatment of acute cerebral infarction and its effect on serum high-sensitivity C-reactive protein (hsCRP) and interleukin (IL).

Methods: One hundred and eighty patients with acute cerebral infarction (ACl) who were on admission from March 2015 to July 2016 participated in this study as research subjects. They were assigned to study group (59 patients) and control group (59 patients). Edaravone and conventional treatment were administered to the control group. In contrast, in addition to conventional treatment, the study group was given edaravone in combination with urinary kallidinogenase. Clinical effects, neurological function and serum IL-17 and hs-CRP levels in the two groups were determined.

Results: The overall response of the study group was significantly higher than that of the control group $(p<0.05)$. Scores in the National Institute of Health stroke scale (NIHSS) were reduced in both groups, and modified Barthel index (MBI) of both groups remarkably increased, when compared to values before treatment. Improvements in NIHSS score and MBI of the study group were higher than those of the control group $(p<0.05)$. Serum IL-17 and hs-CRP levels declined significantly in the two groups ( $p$ $<0.05$ ), but post-treatment serum IL-17 and hs-CRP levels of the study group were significantly reduced, relative to control values $(p<0.05)$. There were no significant differences in incidence of adverse reactions between the two groups.

Conclusion: The use of edaravone in combination with urinary kallidinogenase in the treatment of $\mathrm{ACI}$ can significantly reduce serum IL-17 and hs-CRP levels without inducing severe adverse reactions.
\end{abstract}

Keywords: Edaravone, Urinary kallidinogenase, Acute cerebral infarction

\begin{abstract}
This is an Open Access article that uses a funding model which does not charge readers or their institutions for access and distributed under the terms of the Creative Commons Attribution License (http://creativecommons.org/licenses/by/4.0) and the Budapest Open Access Initiative (http://www.budapestopenaccessinitiative.org/read), which permit unrestricted use, distribution, and reproduction in any medium, provided the original work is properly credited.

Tropical Journal of Pharmaceutical Research is indexed by Science Citation Index (SciSearch), Scopus, International Pharmaceutical Abstract, Chemical Abstracts, Embase, Index Copernicus, EBSCO, African Index Medicus, JournalSeek, Journal Citation Reports/Science Edition, Directory of Open Access Journals (DOAJ), African Journal Online, Bioline International, Open-J-Gate and Pharmacy Abstracts
\end{abstract}

\section{INTRODUCTION}

Acute cerebral infarction (ACl), one of the common cerebrovascular diseases, is a series of pathological and physiological changes induced by cerebral ischemic injury $[1,2]$. Acute cerebral infarction $(\mathrm{ACl})$ has an increasing incidence, with high disability and fatality rates. It has severe effect on the life and health of the affected patients. The key to clinical treatment of acute cerebral infarction is to rapidly relieve cerebral anoxia and ischemia or give thrombolytic 
therapy. The contraindication, indication and window phase of thrombolysis are strict, thus restraining its clinical application and increasing treatment difficulties [3]. Currently, conventional treatment is used in the management of $\mathrm{ACl}$ acute cerebral infarction, but these are not very effective [4].

Edaravone and urinary kallidinogenase are novel drugs which appeared in the market in recent years. As a free radical scavenger, edaravone can effectively eliminate free radicals and inhibit the oxidation of cerebral cells and vascular endothelial cells. Urinary kallidinogenase is a selective cerebrovascular dilator which relaxes blood vessels and improves aerobic level of brain tissues. Edaravone in combination with urinary kallidinogenase acts on multiple pathological steps of cerebral injury induced by acute cerebral infarction, and its effect has been proven in many studies $[5,6]$.

However, not much is known about the effects of edaravone-urinary kallidinogenase combination on inflammatory factors in the treatment of $\mathrm{ACl}$, apart from a few studies [7]. Inflammatory factors are involved in the occurrence and development of inflammatory reactions, and inflammatory reactions play critical roles in the occurrence and development of $\mathrm{ACl}$. This study investigated effectiveness of edaravone-urinary kallidinogenase combination as therapy for $\mathrm{ACl}$, and its effects on serum levels of interleukin (IL)17 and high-sensitivity C-reactive protein (hsCRP).

\section{EXPERIMENTAL}

\section{General characteristics of the study subjects}

One hundred and eighteen patients with $\mathrm{ACl}$ who were on admission between March 2015 and July 2016 were enrolled. The patients met the diagnostic criteria formulated in the $4^{\text {th }}$ National Academic Conference of Cerebrovascular Diseases. They were diagnosed using head CT scan and/or MRI. Patients were excluded if they had recent hemorrhagic disease or bleeding tendency, vital organ, failure, hematological system disorders, mental disease or congenital abnormality in limb function. Moreover, patients who received an altered treatment scheme because of allergy were excluded. The patients were assigned to two groups with the assistance of random number table. There were 59 subjects in each group (control and study groups). The study group had 32 males and 27 females, with mean age of $56.4 \pm 2.7$ years, and there was a mean time interval of $24.5 \pm 9.57 \mathrm{~h}$ between the first dose and onse. In the control group, there were 30 males and 29 females, with a mean age of $55.8 \pm 2.3$ years and a mean interval of $23.6 \pm$ $10.58 \mathrm{~h}$ between the first dosing and onset. Ethical approval was obtained from the Medical Ethics Committee of Binzhou People's Hospital (approval no. WXM20160821BZ), and the guidelines of the Declaration of Helsinki were followed [8].

\section{Therapeutic methods}

The patients were given conventional symptomatic treatment to prevent coagulation and infection, and to improve microcirculation, In addition, the conventional treatment served to maintain water and electrolyte balance, and control blood pressure and glucose. Patients in the control group were given intravenous drip of a mixture containing $30 \mathrm{mg}$ of edaravone (Xi'an Lijun Pharmaceutical Co. Ltd., China; SFDA approval no. H20120042) and $100 \mathrm{~mL}$ of normal saline in addition to conventional treatment, twice a day. Patients in the study group were given intravenous drip of a mixture containing 0.15 PNA of urinary kallidinogenase (Guangdong Techpool Biochemical Pharmaceutical Inc., China; approval number: H20052065), twice a day. The course of treatment of both groups was two weeks.

\section{Observational indexes}

Neurologic impairment in the two groups was evaluated using NIHSS [9] and MBI before and after treatment. Venous blood $(5 \mathrm{~mL})$ was taken from every patient in the fasting state in the morning before and after treatment. Then the blood was subjected to centrifugation at 3000 $\mathrm{rpm}$ at room temperature for $15 \mathrm{~min}$. Serum was separated from the blood, divided into two portions, and preserved at $-70^{\circ} \mathrm{C}$. Levels of IL17 were assayed using ELISA. Serum level of hs-CRP was measured using latex-enhanced immunotransmittance turbidimetry (LEITD). The kits used in the assay were purchased from Shanghai Enzyme-linked Biotechnology Co., Ltd., China, and all the operations strictly followed the kit instructions. Blood and urinary routine indices, hepatic and renal functions, and coagulation function were determined before and after treatment. Electrocardiograph and blood pressure were monitored and recorded. Incidents of untoward effects were recorded.

\section{Determination of therapeutic effects}

A decrease in NIHSS score was taken as the criterion for determination of therapeutic effect [10]. If NIHSS score and grade 0 disabilities decreased by $91-100 \%$, the patient was 
considered as basically cured. If NIHSS score and grades 1 - 3 disabilities decreased by 46 $91 \%$, the patient was considered as significantly progressive. If NIHSS score and grade 4 disabilities decreased $18-46 \%$, it was considered as progressive. A decrease smaller than $18 \%$ was taken as no changes. An increase in NIHSS score and grade 5 disabilities or death were taken as ineffective. Total effectiveness $(\mathrm{T})$ was evaluated using Eq 1.

$T(\%)=\{(A+B+C) / N\} 100$

where $\mathrm{A}, \mathrm{B}, \mathrm{C}$, and $\mathrm{N}$ are no. of cases basically cured, significantly progressive cases, no of progressive cases, and total no. of cases, respectively.

\section{Statistical analysis}

SPSS ver. 21.0 was used in data analysis. Data on enumeration are presented as number or percentage ( $n$, \%). The two groups were compared using Chi-square test. Measurement data are expressed as mean \pm standard deviation (SD) and compared $t$-test. Values of $p$ $<0.05$ implied significant differences.

\section{RESULTS}

\section{Clinical effectiveness}

The total effectiveness in the study group was $94.9 \%(56 / 59)$, which was remarkably higher than $78.0 \%(46 / 59)$ seen in the control group $\left(X^{2}\right.$ $=4.176, p<0.05$, Figure 1).

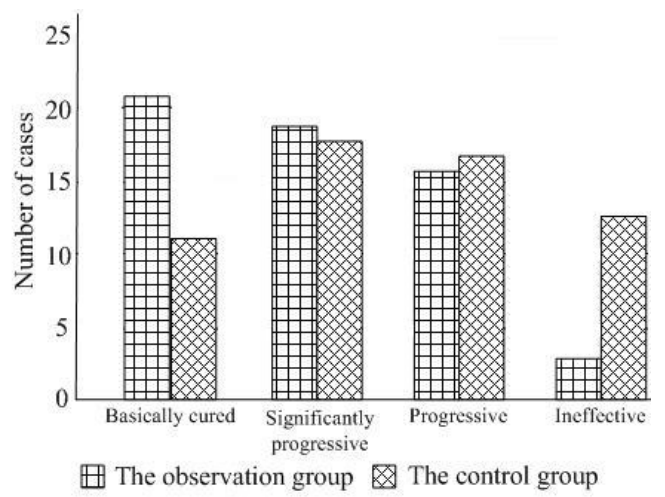

Figure 1: Clinical effectiveness of the treatments

\section{Changes in neurological function}

There were significant increases in NIHSS scores in the study and control groups after treatment, relative to corresponding pretreatment scores $(p<0.05$, Table 1$)$. However, the $\mathrm{MBI}$ scores became significantly lower after treatment. The variations in the two indices in the study group were larger than the variations in the control group

Table 1: NIHSS and $\mathrm{MBI}$ scores before and after treatment (mean $\pm S D$, point)

\begin{tabular}{lcc}
\hline Group & NIHSS & MBI \\
\hline Study group & $14.71 \pm 2.49$ & $4.63 \pm 0.31$ \\
& $4.52 \pm 0.45^{\text {\#\# }}$ & $8.95 \pm 0.57^{\text {\#\# }}$ \\
Control group & $14.39 \pm 2.44$ & $4.66 \pm 0.34$ \\
& $5.59 \pm 0.27$ & $7.56 \pm 0.42$ \\
\hline${ }^{*} P<0.05$, compared to before treatment; \#p < 0.05, \\
compared to control
\end{tabular}

\section{Serum levels of IL-17 and hs-CRP}

The differences in pre-treatment serum levels of IL-17 and hs-CRP between the two groups were not statistically significant $(p>0.05)$. However, there were significant differences in IL-17 and hsCRP levels between both groups $(p<0.05)$. The study group had significantly lower serum levels of IL-17 and hs-CRP than the control group ( $p<$ 0.05 , Table 2).

Table 2: Serum levels of IL-17 and hs-CRP before and after treatment

\begin{tabular}{lcc}
\hline Group & IL-17 (ng/L) & $\begin{array}{c}\text { hs-CRP } \\
\text { (mg/L) }\end{array}$ \\
\hline Study group & $105.07 \pm 32.16$ & $14.20 \pm 3.18$ \\
& $34.81 \pm 11.26^{\text {*\# }}$ & $4.65 \pm 1.35^{\pi \#}$ \\
Control group & $107.22 \pm 31.35$ & $14.18 \pm 3.27$ \\
\hline
\end{tabular}

${ }^{*} p<0.05$, compared to before treatment; $\# p<0.05$, compared to control

\section{Adverse reactions}

None of the patients in the two groups had severe impairment of the liver, heart and kidney. Moreover, blood routine indices of the two groups had no apparent changes. Only one patient in the study group blushed and felt feverish when receiving being intravenous drip of urinary kallidinogenase, but recovered after the infusion was slowed down.

\section{DISCUSSION}

Cerebral infarction is induced by cerebral ischemia caused by cerebrovascular occlusion. Neurological function deteriorates several hours or days after the attack. Therefore treatment of cerebral infarction usually includes cerebral protection, alleviation of neurological function and acceleration of neurological function recovery [11]. Currently, cerebral infarction is treated with multiple drugs, but the effectiveness of those drugs are not adequate. Thus, there is need to improve therapeutic effect by developing new drugs.

Trop J Pharm Res, December 2018; 17(12): 2479 
In the present study, edaravone in combination with urinary kallidinogenase was used to treat acute cerebral infarction, with favourable results. In many animal studies, urinary kallidinogenase has been proven effective in promoting vascularization in ischemic tissues, relieving inflammation and nerve cell apoptosis, and increasing hemoperfusion volume in local tissues. Urinary kallidinogenase can be used in the treatment of cerebral stroke, and its main component is human urinary kallidinogenase. It can effectively inhibit platelet aggregation and relieve intracranial blood steal phenomenon $[12,13]$. It improves blood circulation, stimulates vascular endothelial cells to secrete NO, eliminates free radicals, inhibits oxidative stress and IL-6, dredges occlusive vessels, and accelerates neovascularization [14].

Edaravone is a brain-protective agent which can prevent reduction of cerebral blood flow volume around infarcts and assist urinary kallidinogenase to eliminate free radicals, inhibit lipid peroxidation, and relieve impairment of vascular endothelial cells and brain cells $[15,16]$. The results of this study have demonstrated superior clinical effect in the combination treatment group, and the improvement of NIHSS score and $\mathrm{MBI}$ was better in the study group than in control group. This indicates that edaravoneurinary kallidinogenase combination can effectively reduce neurological impairment.

Inflammatory reaction is also an important factor that influences the onset of acute cerebral infarction. When local cerebral ischemia happens, many oxygen free radicals are generated. These induce the activation of immune system after white cells enter ischemic tissues; then pro-inflammatory factors are released to mediate inflammatory reactions, which aggravate the apoptosis of nervous cells in ischemic sites. Inflammatory reaction may promote the formation and spread of atherosclerotic plaques which are one of the leading independent risk factors for acute cerebral infarction; most patients with acute cerebral infarction have atherosclerotic plaques [17]. It is known that hs-CRP is one of the common markers of inflammatory reaction. Studies have shown that patients with acute cerebral infarction experience sudden increases in hs-CRP levels [18]. This is consistent with the condition of the patients in the current study. As an inflammatory reaction factor, IL-17 stimulates epithelial cells, endothelial cells, fibroblast and keratinocytes to release cytokines.
Moreover, IL-17 can aggravate inflammatory reaction through up-regulation of cytokines [19]. In addition, IL-17 upregulates the production of intercellular adhesion molecule-1 (ICAM-1) and promotes the secretion and release of inflammatory reaction-related factors such as tumor necrosis factor (TNF)- $\alpha, \quad \mathrm{IL}-6$ and IL-8 which aggravate inflammatory reactions and cerebral injury [20]. The current study indicate that the patients in the study group had remarkably lower serum levels of IL-17 and hsCRP than the control group patients. This suggests that edaravone in combination with urinary kallidinogenase can effectively inhibit inflammatory reactions and enhance therapeutic effectiveness.

\section{Limitations of the study}

The source of the cases was limited; therefore, it cannot be ruled out that the conclusions of this article may be affected by some biases. Moreover, the patients were not followed up for a long time. To validate the findings in this study, multi-center clinical trials with larger sample sizes need to be carried out in the future

\section{CONCLUSION}

Edaravone in combination with urinary kallidinogenase is effective in treating cerebral infarction. The therapy can relieve neurological impairment, inhibit inflammatory reaction, and improve prognosis. The number of cases included in this study was small. To ensure the preciseness of results, studies with a larger sample size are needed in the future. A follow-up study is also necessary.

\section{DECLARATIONS}

\section{Conflict of Interest}

No conflict of interest associated with this work.

\section{Contribution of Authors}

We declare that this work was done by the authors named in this article and all liabilities pertaining to claims relating to the content of this article will be borne by the authors. Xin-Min Wang, Li Zhu and Ying-Jun Sun: Study design, data collection and analysis. Xiao-Ying Liu, ShuGang Dong, Jing Han and Jin-Ming Ji: Manuscript preparation, drafting and revising. Xin-Min Wang: Review and final approval of manuscript. 


\section{REFERENCES}

1. Emanueli C, Graiani G, Salis MB, Gadau S, Desortes E, Madeddu P. Prophylactic gene therapy with human tissue kallikrein ameliorates limb ischemia recovery in type I diabetic mice. Diabetes 2004; 53(14): 1096-1113.

2. Xia CF, Yin H, Borlongan CV, Chao L, Chao J. Kallikrein gene transfer protects against ischemic stroke by promoting glial cell migration and inhibiting apoptosis. Hypertension 2006; 43(15): 452-459.

3. Li B. Observation of effect of edaravone in combination with urinary kallidinogenase in the treatment of acute cerebral infarction. Chin J Clin Rational Drug Use 2016; 9(5): 32-33.

4. Wang AH. Clinical observation of edaravone in treatment of acute cerebral infarction. J Tradit Chin Med Univer Hunan 2010; 30(12): 50-52.

5. Yang J. To explore the clinical effect of edaravone combine with urinary kallidinogenase treatment acute cerebral infarction. Chin Contin Med Edu. 2016; 7(19): 161-162.

6. He $Q$, Wu YQ. Edaravone combined with urinary kallidinogenase for acute cerebral infarction. Prog Mod Biomed 2010; 10(24): 4733-4757.

7. Hosaka K, Hoh BL. Inflammation and cerebral aneurysms. Translat Stroke Res 2014; 5(2): 190-198.

8. Declaration of Helsinki. The 59th World Medical Conference, 2008.

9. The 4th National Academic Conference of Cerebrovascular Disease. Scoring Standards for the Clinical Nerve Function Deficiency of Patients with Cerebral Stroke (1995). Chin J Neurol 1996; 29(6): 381 383.

10. Naess H, Kurtz M, Thomassen L, Waje-Andreassen U. Serial NIHSS scores in patients with acute cerebral infarction. Acta Neurol Scand 2016; 133(6): 415-420.
11. Yu CX, Yang MH. Risk factors for aggravation of cerebral infarction at acute stage. J Baotou Med Coll 2016; 32(8): 42-43.

12. Xiao J, Li F, Gu DX. Observation of effect of in the treatment of acute cerebral infarction. Chin J Nerv Mental Dis 2008; 34(1): 54-55.

13. Shi $Q D$, Zhang JF, Zhang $P B$, Wang $X$, Chen $X L$, Tian $Y M$, Yang $P B$, Zhang JS, Liu Y. Effects of human urinary kallikrein on regional cerebral blood flow in penumbra following focal cerebral ischemia in rats. Chin J Nerv Mental Dis 2008; 34(7): 413-416.

14. Wang Z, You SL. Effects of urinary kallidinogenase on collateral circulation for patients with acute cerebral infarction of anterior circulation. J Clin Res 2015; 32(2): 325-327.

15. Miao J, Deng F, Zhang Y, Xie HY, Feng JC. Exogenous human urinary kallidinogenase increases cerebral blood flow in patients with acute ischemic stroke. Neurosciences 2016; 21(2): 126-130.

16. Kimura K, Aoki J, Sakamoto Y, Kobayashi K, Sakai K, Inoue $T$, Iguchi $Y$, Shibazaki $K$. Administration of edaravone, a free radical scavenger, during $t-P A$ infusion can enhance early recanalization in acute stroke patients - a preliminary study. J Meurol Sci 2012; 313(1-2): 132-136.

17. Verweij SL, Fm VDV, Stroes ES. Novel directions in inflammation as a therapeutic target in atherosclerosis. Curr Opinion Lipidol 2015; 26(6): 580-585.

18. Zhou $Y$, Han W, Gong D, Man C, Fan Y. Hs-CRP in stroke: A meta-analysis. Clin Chim Acta 2016; 453: 21 27.

19. Waisman A, Hauptmann J, Regen T. The role of IL-17 in CNS diseases. Acta Neuropathol 2015; 129(5): 1-13.

20. Swardfager W, Herrmann N, Andreazza AC, Swartz RH, Khan MM, Black SE, Lanctôt KL. Poststroke neuropsychiatric Symptoms: relationships with IL-17 and oxidative stress. Biomed Res Int 2014; 2014(1): 12851289. 